\title{
The Management of Metastatic Thyroid Carcinoma: An Initial Presentation with Bony Metastasis
}

\author{
Tony Y. Eng ${ }^{1,2 *}$, Samantha Litchke ${ }^{1}$, Aidnag Z. Diaz ${ }^{3}$, Join Y. Luh ${ }^{4}$ \\ ${ }^{1}$ Department of Radiation Oncology, University of Texas Health Science Center, San Antonio, USA; ${ }^{2}$ Cancer Therapy and Research \\ Center, San Antonio, USA; ${ }^{3}$ University Radiation Medicine, Woman's Board Center for Radiation Therapy, Chicago, USA; ${ }^{4}$ Radiation \\ Oncology, St. Joseph Hospital, Eureka, USA. \\ Email: Tyeng@pol.net
}

Received December $18^{\text {th }}$, 2010; revised July 29 ${ }^{\text {th }}$, 2011; accepted August $8^{\text {th }}, 2011$.

\begin{abstract}
Introduction: Metastatic thyroid carcinoma presenting at the initial time of diagnosis is uncommon and the prognosis is unclear. Long term survival rates are variable ranging from $13 \%$ to $100 \%$. This case report is presented to illustrate potential management and lend statistical power to future analysis of the correct treatment planning, mortality rates, and prognostic indications for an uncommon presentation of thyroid cancer. Case Presentation: This patient is a 63 year old female who presented with new onset of progressive right hip pain. She was treated with a cortisone injection for presumed osteoarthritis but did not improve. Physical exam at the time was pertinent for a body mass index (BMI) of 38.4, mild systolic hypertension, difficulty walking secondary to the right hip pain, limited range of motion at the hip, and fullness of right thyroid gland with no palpable nodules. Laboratory evaluation including thyroid function tests was normal. However, CT and MRI scans revealed a $6.5 \mathrm{~cm} \times 5 \mathrm{~cm}$ osteolytic expansive lesion on the right iliac crest with a soft tissue mass. In addition, an enlarged right thyroid lobe and small nodular densities in the lungs suspicious for metastatic disease were noted. A fine needle aspiration of the right ileum revealed metastatic follicular adenocarcinoma consistent with a thyroid primary. The patient was not a surgical candidate due to the extent of disease. She received 37.5 Gy to both the right iliac crest mass and the neck to include the thyroid lesion, followed by iodine-131 ablation and bisphosphonate therapy. Her disease was stable on her last follow up at 48 months. Conclusions: Further studies identifying independent variables such as age of the patient, site and extent of the disease and histology of the tumor are needed to help determine the true prognosis and proper management of patients with this presentation. The optimal treatment with potential chance for cure in patients with metastatic thyroid carcinoma has yet to be elucidated.
\end{abstract}

Keywords: Thyroid Cancer, Metastatic Disease, Radiation Therapy

\section{Introduction}

Thyroid carcinoma is relatively uncommon, accounting for $2 \%$ of all cancers. In general, thyroid cancers have a low mortality rate, an excellent prognosis and are more common in women than men. Eighty-five percent of all thyroid cancers are of the well-differentiated type and include papillary, follicular, and Hurthle cell carcinoma. Differentiated thyroid carcinoma is one of the most curable cancers. The less common and poorer prognosis types of thyroid cancer include medullary, anaplastic, lymphoma, and metastatic disease [1]. The metastatic group includes those with distant metastases at initial presentation and those with metastases occurring after treatment. Although this group accounts for less than $10 \%$ of the thyroid cancers, distant metastatic disease unfortunately represents the most common cause of thyroid cancer-related deaths with a wide range of survival [2,3]. Only $1 \%-4 \%$ of people with thyroid carcinomas present with distant metastatic disease at the time they are initially diagnosed [3]. This paper will present a case report on an individual with distant metastases at the time of diagnosis. The true prognosis is difficult to assess and optimal management remains unclear. This case report is presented to lend statistical power to future analyses of optimal treatment planning, mortality rates, and prognostic factors for an uncommon presentation of thyroid cancer. 


\section{Case History}

A 63 year old female nurse presented in October of 2006 with recent onset progressive right hip pain. She was treated for osteoarthritis but did not improve and was then treated with a cortisone injection. Past medical history is pertinent for hypertension for which she takes hydrochlorothiazide and lisinopril. Past surgical history includes a total abdominal hysterectomy and salpingooophorectomy in 1982 for benign reasons, bladder suspension in 1987 and right breast lumpectomy in 2001 with benign pathology. Family history is positive for basal cell carcinoma of the skin, strokes, COPD, and a maternal aunt with breast cancer. She is married, has three children, and has smoked 1 to 2 cigarettes per day for twenty years but recently quit. She does not drink alcohol or use illicit drugs. The review of systems is positive for weakness, fatigue and the hip/leg pain. The patient denies any hot or cold intolerance or hair loss. Physical exams during her visits were pertinent for a BMI of 38.4 (217 pounds, 5 foot 3 inches tall), mild systolic hypertension, difficulty walking secondary to the right hip pain, limited range of motion at the hip , and fullness of right thyroid gland without palpable nodules. Laboratory evaluation included normal complete blood count, urinalysis and liver function tests, as well as, elevated blood urea nitrogen of 36 and a creatinine of 1.6 with normal thyroid function tests. Imaging studies including CT scan and MRI revealed small nodular densities in the lungs bilaterally suspicious for metastatic disease, an enlarged right thyroid lobe, and a $6.5 \mathrm{~cm} \times 5 \mathrm{~cm}$ osteolytic expansive lesion invading the right iliac crest with a soft tissue mass (Figure 1). A nuclear medicine bone scan revealed increased activity on the left proximal femur which surrounded a soft tissue mass and increase activity in the thoracolumbar spine and $6^{\text {th }}$ rib. A fine needle aspiration of the right ileum revealed metastatic follicular adenocarcinoma consistent with a thyroid primary. The patient's thyroglobulin (TG) level was done and was elevated at 34,922 $\mathrm{ng} / \mathrm{mL}$.

The patient was not deemed a surgical candidate due to the extent of disease and was referred for evaluation by radiation oncology. The patient received 37.5 Gy to the right iliac crest and the left proximal femur, as well as, a palliative course of 37.5 Gy to the thyroid lesion (Figure 2). Medical oncology did not see any role for chemotherapy at this juncture but recommended iodine131 ablation and bisphosphonates (zoledronic acid) after the palliative radiotherapy was completed.

On her last follow up at 48 months, she still had mild chronic baseline bone pain which was well controlled with oral analgesics. She remained ambulatory and was able to do routine daily activities. Her disease was stable

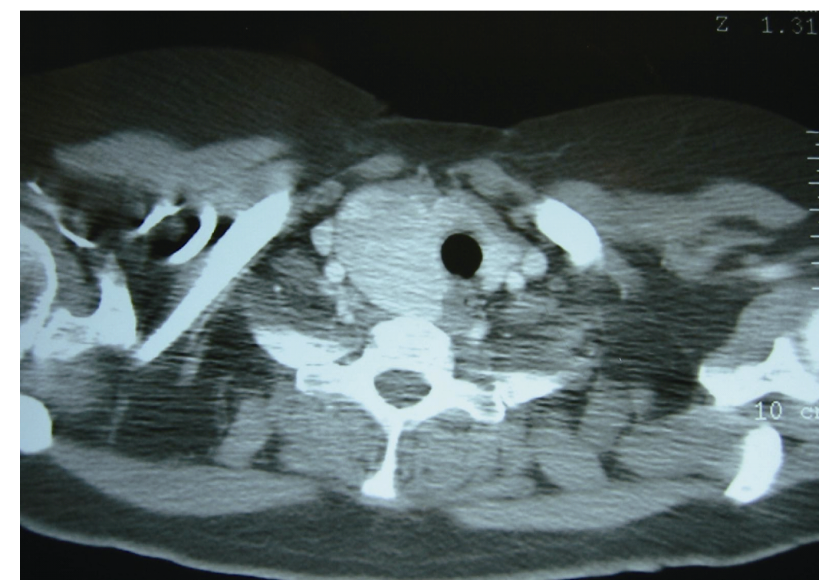

(a)

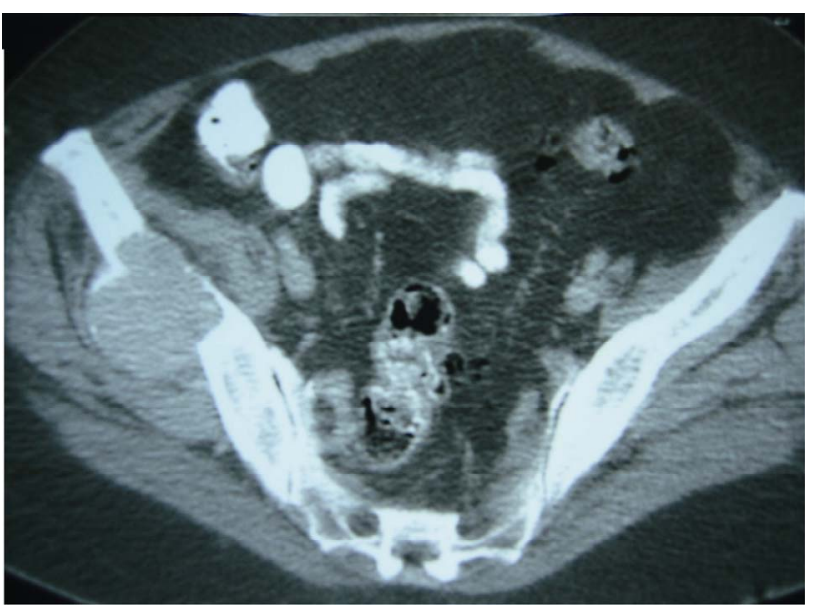

(b)

Figure 1. Computed tomography (CT) scan showing a large thyroid mass displacing adjacent vasculature (a). An invasive soft tissue mass infiltrating into the right iliac bone (b).

radiographically. Her last follow-up TG level was 732.

\section{Discussion}

Treatment planning, mortality rates and prognostic factors are unclear in metastatic thyroid carcinoma because it is uncommon. Up to the present time radioiodine was the only available systemic modality to treat patients with metastatic disease.

Durante et al. [3] looked at the benefits and limits to radioiodine therapy in the long term outcome of 444 patients with distant metastatic disease from well differentiated thyroid carcinomas. They concluded that I-131 treatments are highly effective in younger patients with I131 uptake and with small metastases. They recommended treatment until the disappearance of any uptake or until a total of $22 \mathrm{GBq}$ (595 mCi) has been given. Patients who are older than 40, have a large extent of metastasis, poorly differentiated or no I-131 uptake may be 


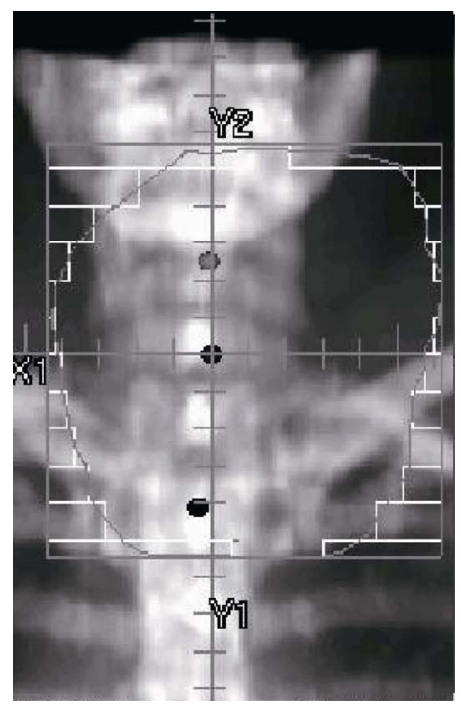

(a)
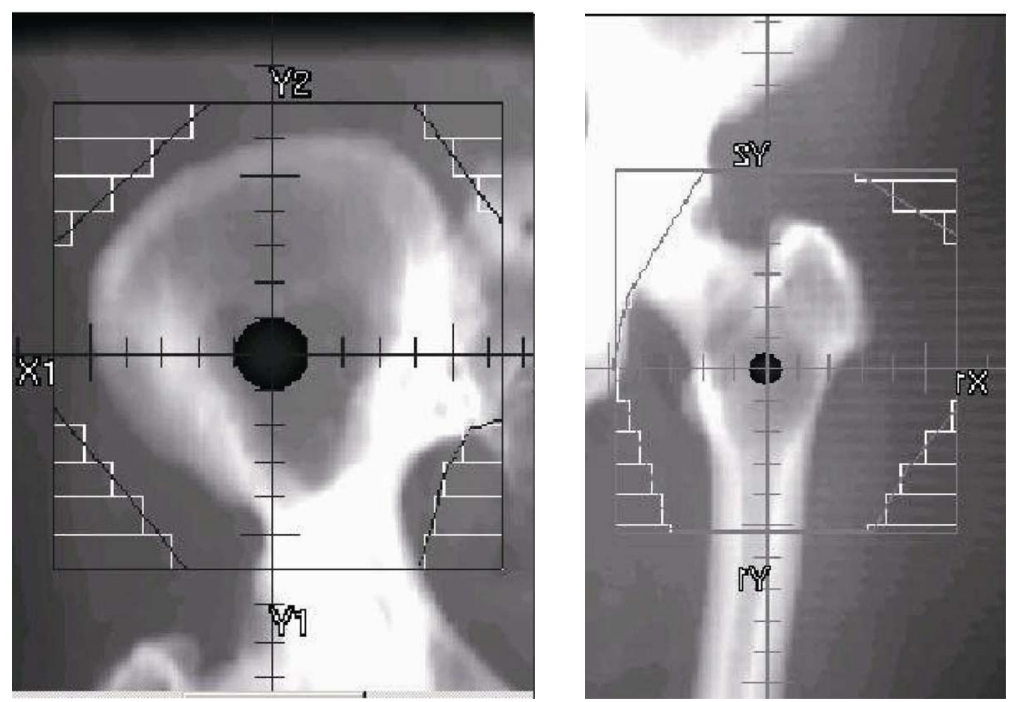

(b)

Figure 2. Treatment Fields: the masses in the thyroid (a). Right iliac bone and left proximal femur (b) are treated with two opposing beams.

candidates for therapeutic trials with molecular targeted therapies [such as sorafenib and sunitinib]. This retrospective study stratified patients into three groups according to the age at detection of metastases. The 10-year survival rate was $14 \%$ in the 266 patients older than 40 with macronodular lung metastases or multiple bone metastases. In contrast, the survival rate at 10-years in the 113 patients younger than 40 with metastases that were not visible on radiographs or that were micronodular was 95\%. For the remaining 62 patients who were either older than 40 and had metastases that were not visible or were micronodular or who were younger than 40 and had macronodular lung metastases, the 10 -year survival rate was $64 \%$ [3].

At the Memorial Sloan-Kettering Cancer Center, Margo et al. [2] performed a retrospective review of 242 subjects with distant metastases from differentiated thyroid cancer available in their thyroid cancer research data base. The study found that at the age of 45 years or greater, metastatic sites other than lung or bone only, and symptoms at the time of diagnosis, are associated with poorer outcomes. Their policy is to treat patients with distant disease with completion thyroidectomy when necessary, followed by ablative doses of I-131. They emphasize the importance of evaluating on an individual basis before predictions of survival are determined.

Bennbassat et al. [4] at the Rabin Medical Center in Tel Aviv, Israel studied the long term outcome of patients with DTC (differentiated thyroid cancer) and distant metastasis. Review of medical records of 44 patients with DTC and distant metastasis led him to conclude that complete resection of the thyroid gland followed by high dose adjuvant radioactive iodine therapy and palliative external beam radiation therapy as needed was associated with improved survival, 88\% 5 year and 75\% 10 year survival. Doses of iodine ablation varied from mean doses of $444 \mathrm{mCi}$ for patients with lung metastases to $638 \mathrm{mCi}$ for patients with bone metastasis.

At the Royal Marsden Hospital in the United Kingdom, Haq et al. [5] retrospectively evaluated 111 patients with DTC who presented with distant metastasis. They concluded that in these patients age over 70 years, poorly differentiated tumors, and Hurthle cell histology were poor prognostic indicators. The prognosis of patients with DTC, even in this subset of patients with poor prognosis, has significantly improved in the modern era, from 1991 to 2002 with a cause specific survival of over $80 \%$ at 5 years. The authors attribute this to the combination of total thyroidectomy, radionuclide ablation, localized radiotherapy, when indicated, and TSH suppression. They advocate an aggressive multidisciplinary approach.

Thus, for metastatic disease, the 2011 update of the National Comprehensive Cancer Network (NCCN) Guidelines make several recommendations depending on the sites of metastases [6]. Patients are advised to stay on levothyroxine to suppress TSH levels. For symptomatic or asymptomatic bone metastases in weight bearing regions, orthopedic surgical palliation, I-131 treatment (for positive radioiodine imaging), and external beam radiation therapy are acceptable therapeutic options. Intravenous bisphosphonate therapy (zolendronate or pamidronate) can be considered for other symptomatic bone metastases.

For central nervous system (CNS) metastases, the 
NCCN panel recommends neurosurgical resection, radioiodine treatment (for positive radioiodine imaging), and/or image-guided radiation therapy should be considered. Solitary CNS lesions should be treated with either neurosurgical resection or stereotactic radiosurgery. Other sites of metastases should be treated with surgical resection, radiation therapy, and/or I-131 (for tumors that are radioiodine avid).

In terms of systemic therapy, the NCCN guidelines recommend clinical trials for non-radioiodine avid tumors. Novel molecular targeted therapies, or traditional cytotoxic systemic therapy (doxorubicin alone or in combination with other agents) can be considered if a trial is not available. A list of current clinical trials can be found at the American Thyroid Association's clinical trials website at http://www.thyroidtrials.org [7].

\section{Conclusions}

Further studies identifying independent variables such as age of the patient, site and extent of disease, and histology of the tumor are needed to help determine the true prognosis and optimal management of patients with metastatic thyroid cancer at presentation. Currently, there is no consensus in the management of metastatic thyroid cancer, especially in the setting of I-131 resistance. Palliative radiation therapy helps control pain and decrease the risk of impending fracture. Systemic chemotherapy may lead to occasional response, but overall, results have been disappointing. However, future studies are needed to determine the optimal optimal treatment and potential chance for cure in patients with distant metastatic thyroid carcinoma.

\section{Consent}

Informed written consent was obtained from the patient for publication of the manuscript and figures.

\section{REFERENCES}

[1] S. Lai, Y. Susan, J. Mandel and R. S. Weber, "Disorders of the Thyroid Gland,” In: C. W. Cummings, B. H. Haughey, J. R. Thomas, L. A. Harker, K. Robins, P. W. Flint, D. Schuller and R. M. Cummings, Eds., Otolaryngology: Head and Neck Surgery, Mosby Pub, Inc., 2005.

[2] S. Margo, A. Stojadinovic, A. Nissan, R. A. Ghossein, S. Freedman, M. F. Brennan, J. P. Shah and A. R. Shaha, "Prognostic Indicators of Outcomes in Patients with Distant Metastases from Differentiated Thyroid Carcinoma," Journal of the American College of Surgeons, Vol. 197, No. 2, 2003, pp. 191-197.

doi:10.1016/S1072-7515(03)00332-6

[3] C. Durante, N. Haddy, E. Baudin, S. Leboulleux, D. Hartl, J. P. Travagli, B. Caillou, M. Ricard, J. D. Lumbroso, F. De Vathaire and M. Schlumberger, "Long-Term Outcome of 444 Patients with Distant Metastases from Papillary and Follicular Thyroid Carcinoma: Benefits and Limits of Radioiodine Therapy,” Journal of Clinical Endocrinology \& Metabolism, Vol. 91, No. 8, 2006, pp. 2892-2899. doi:10.1210/jc.2005-2838

[4] C. A. Benbassat, S. Mechlis-Frish and D. Hirsch, "Clinicopathological Characteristics and Long-Term Outcome in Patients with Distant Metastases from Differentiated Thyroid Cancer," World Journal of Surgery, Vol. 30, No. 6, 2006, pp. 1088-1095. doi:10.1007/s00268-005-0472-4

[5] M. Haq and C. Harmer, "Differentiated Thyroid Carcinoma with Distant Metastases at Presentation: Prognostic Factors and Outcome," Clinical Endocrinology, Vol. 63, No. 1, 2005, pp. 87-93. doi:10.1111/j.1365-2265.2005.02304.x

[6] National Comprehensive Cancer Network, "NCCN Clinical Practice Guidelines in Oncology: Thyroid Carcinoma,” 2011. http://www.nccn.org/professionals/physician_gls/f_guidel ines.asp

[7] American Thyroid Association, “Thyroid Clinical Trials,” Falls Church, 2011. http: //thyroidtrials.org/ 